\title{
¿Cómo alcanzó Costa Rica la incorporación social y laboral?
}

\author{
Juliana Martínez Franzoni y Diego Sánchez-Ancochea
}

\section{Resumen}

En 1980, justo antes de la crisis de la deuda y en contraste con la experiencia del resto de América Latina, prácticamente toda la población costarricense contaba con empleos formales y servicios sociales de calidad. Para explicar esta doble incorporación social y laboral, en este artículo se cuestionan el papel de la distribución de la tierra, la calidad de las instituciones públicas y la democracia. En su lugar, nos enfocamos en el proceso de construcción del Estado mediante el cual una pequeña élite emergente de empresarios y profesionales urbanos, nutriéndose de ideas internacionales y adaptándolas adecuadamente, utilizó la política pública para manejar el conflicto social y ampliar sus propias oportunidades económicas. Más allá de Costa Rica, este análisis tiene especial relevancia en el marco del creciente énfasis en la economía política de las políticas públicas y de la aún insuficiente atención a las élites que participan en su diseño.

\section{Palabras clave}

Condiciones económicas, condiciones sociales, bienestar social, empleo, seguridad social, política social, Costa Rica

Clasificación JEL

$$
\text { 13, } 054
$$

Autores

Juliana Martínez Franzoni es Catedrática del Instituto de Investigaciones Sociales de la Universidad de Costa Rica. juliana.martinez@ucr.ac.cr

Diego Sánchez-Ancochea es Profesor Titular de la Universidad de Oxford (Reino Unido) y Director del Centro Latinoamericano de la misma Universidad. diego.sanchez-ancochea@ qeh.ox.ac.uk 


\section{Introducción ${ }^{1}$}

El bienestar de las personas depende simultáneamente de su adecuada incorporación al mercado laboral y de su protección frente a la enorme volatilidad de ese mismo mercado. Por un lado, las personas necesitan contar con un ingreso estable seguro para incrementar su nivel de consumo y así satisfacer las demandas del hogar. Como Banerjee y Duflo (2011, pág. 227) enfatizan en su libro más reciente, Poor Economics: A Radical Rethink of the Way to Fight Poverty, en donde sea que pregunten, el sueño más común de los pobres es que sus hijas e hijos sean funcionarios del gobierno, lo que sugiere un deseo de estabilidad; esto demuestra la importancia de los trabajos de calidad. Por otro lado, las personas también desean recibir ciertos servicios independientemente del mercado como parte de sus derechos ciudadanos y como forma de asegurarse frente a las crisis y la volatilidad externa.

Para explorar este doble objetivo de la política pública - cuya urgencia han subrayado instituciones como el Instituto de Investigaciones de las Naciones Unidas para el Desarrollo Social (véase UNRISD, 2010) - hemos propuesto los conceptos de incorporación laboral e incorporación social (Martínez Franzoni y Sánchez-Ancochea, 2012)². Por incorporación nos referimos a la participación de las personas en un período histórico largo en dos instituciones clave para la asignación de los recursos: los mercados de trabajo formal y los servicios públicos sociales. La incorporación laboral se refiere al acceso de las personas al intercambio monetario de forma estable, lo cual requiere, a su vez, la creación de un número suficiente de trabajos formales y bien remunerados privados y públicos. La incorporación social se produce cuando las personas son capaces de asegurar su bienestar independientemente del nexo monetario ${ }^{3}$. La noción de doble incorporación a la que se hace referencia en este artículo es, por lo tanto, normativa: no se refiere a cualquier participación en los mercados de trabajo y en los servicios sociales, como la que necesariamente trae consigo el proceso de urbanización, sino al logro de un nivel mínimo monetario, de empleo y protección social, y de servicios para la mayoría de la población.

Desafortunadamente, pocos países del Sur han logrado de forma simultánea la incorporación social y laboral. En América Latina, por ejemplo, la incorporación laboral fue incompleta y la social estuvo segmentada durante la mayor parte del siglo XX. En el período que siguió a la Segunda Guerra Mundial, la baja productividad agrícola conllevó una rápida migración a zonas urbanas donde las nuevas industrias de capital intensivo no lograron crear suficientes empleos. Los niveles de informalidad y desempleo permanecían altos (aunque en menor medida en la Argentina, Chile y el Uruguay que en el Brasil o los países andinos), y en 1980 dos quintos de los empleos latinoamericanos eran informales (Tokman, 2001). La incorporación social reflejó la segmentación ocupacional bajo esquemas contributivos organizados por actividad laboral en torno al empleo formal, que excluían a la población pobre (Seekings, 2008). A inicios de la década de 1970, por ejemplo, la seguridad social chilena contaba con 160 programas distintos con beneficios asimétricos para diferentes grupos ocupacionales (Mesa-Lago, 1978). Funcionarios públicos, profesionales y otros trabajadores

1 Este artículo se basa en el argumento presentado en el libro Good Jobs and Social Services: How Costa Rica Achieved the Elusive Double Incorporation (Martínez Franzoni y Sánchez-Ancochea, 2013a), que no ha sido publicado en español.

2 En el uso de la noción de incorporación nos precede una rica tradición de investigación social. En su ya clásico estudio, Collier y Collier (1991) explican cómo la incorporación - en su caso, a la arena política - reemplazó la represión de la clase trabajadora como actor político.

3 Reygadas y Filgueira (2010) argumentan que América Latina enfrenta actualmente una segunda crisis de incorporación social. En lugar de la clase trabajadora formal que lideró la crisis de incorporación previa, ahora los grandes protagonistas son los millones de trabajadores y trabajadoras informales y carentes de servicios mínimos. La ola de partidos de izquierda y de centroizquierda en el gobierno refleja, en parte, la demanda de incorporación de esta población. 
urbanos formales se incorporaron primero a la seguridad social y gozaron de beneficios mayores que los percibidos por los trabajadores independientes e informales (Filgueira, 2005). En la Argentina, los programas de seguridad social "para grupos menos influyentes y organizados, particularmente trabajadores rurales y domésticos, no lograron sumar afiliados y los beneficios que se ofrecieron fueron usualmente mínimos" (Lewis y Lloyd-Sherlock, 2009, pág. 116).

Costa Rica fue, sin embargo, una excepción no solo en la región sino en todo el mundo en desarrollo: entre 1950 y 1980 el desempleo y el subempleo fueron bajos, el sector formal creció de forma sostenida y se expandió un conjunto de políticas sociales universales ${ }^{4}$. Hacia 1980, la mayoría de los costarricenses tenían acceso a trabajos relativamente bien remunerados y a servicios de salud, educación y pensiones de alta calidad (Sandbrook y otros, 2007). El rendimiento costarricense fue, en algunos aspectos, mejor incluso que el de los países de Asia Oriental. Si bien estos experimentaron un "milagro" laboral y económico, tuvieron a la vez un nivel de gasto social bajo y una red de seguridad social pública poco desarrollada (Goodman y Peg, 1996), y la protección social organizada principalmente en torno a las empresas dejó fuera a una gran proporción de la población (OIT, 2007).

¿Qué explica el éxito de Costa Rica? La incorporación laboral y social a largo plazo fue menos el resultado de una ideología particular y más la consecuencia de las preferencias económicas de una nueva élite cuyo objetivo era la construcción del Estado. Una clase emergente de pequeños y medianos empresarios y profesionales urbanos agrupados en torno al Partido Liberación Nacional (PLN) adquirió protagonismo y recurrió al Estado para atender asuntos tan concretos como obtener crédito para expandir sus oportunidades económicas. A través de la adaptación de ideas internacionales -una variable que por lo general es olvidada en los debates de economía política- estos actores también utilizaron la política pública para aminorar los conflictos sociales y debilitar a la oposición.

De esta forma, el análisis que aquí presentamos va más allá del caso costarricense y contribuye a algunos de los debates actuales más significativos en economía política. Por un lado, a raíz de los trabajos de Acemoglu, Robinson y colaboradores, en la economía convencional se presta una atención creciente al papel del poder de las élites y su influencia en el entramado institucional (Acemoglu y Robinson, 2012; Amsden, Di Caprio y Robinson, 2012). Sin embargo, en pocos de esos trabajos se aborda adecuadamente quiénes conforman las élites y para qué necesitan al Estado. En este artículo, en cambio, destacamos la importancia de aquellos que lideran el proceso de construcción estatal y de las interacciones entre la élite, la burocracia y las ideas internacionales. Por otro lado, al considerar tanto la importancia de estos grupos como su relación con el Estado es posible entender mejor cuáles son las posibilidades y las mejores estrategias para crear nuevos pactos para la igualdad y alcanzar la doble incorporación en América Latina, uno de los objetivos centrales del trabajo reciente de la Comisión Económica para América Latina y el Caribe (CEPAL) (2014).

A continuación, en la segunda sección, discutimos las explicaciones disponibles sobre el éxito de Costa Rica en asegurar la doble incorporación social y laboral entre 1950 y 1980 y sus limitaciones. En la tercera sección de este artículo presentamos un argumento basado en el papel que la élite emergente tuvo en la construcción del Estado. En la cuarta sección analizamos brevemente la validez de las dos variables clave aquí presentadas para explicar los problemas que Costa Rica está teniendo para sostener la doble incorporación desde los años ochenta. Concluimos señalando las implicaciones teóricas y políticas para el presente.

\footnotetext{
4 En su análisis, Pribble (2011) ubica a Costa Rica, junto con la Argentina, Chile y el Uruguay, como país con un alto desempeño en la prevención y el manejo de riesgos. Sin embargo, en su análisis se concentra en el presente de forma exclusiva y no desagrega la incorporación dispar de los grupos sociales dentro de cada país. Sandbrook y otros (2007), por otro lado, subrayan la excepcionalidad costarricense y ubican a este país como el único ejemplo de socialdemocracia en América Latina antes de la década de 1990.
} 


\section{Las explicaciones disponibles del éxito costarricense}

Las explicaciones predominantes del éxito de Costa Rica giran en torno a la existencia de patrones relativamente equitativos de distribución de la tierra y del ingreso que ya desde la Colonia habrían creado las condiciones para la posterior consolidación de la democracia y la creación de capacidades estatales. A pesar de su nombre, Costa Rica carecía de recursos naturales y de una fuerza de trabajo indígena grande, y estaba lejos del centro político español ubicado en Guatemala. Por ello, la distribución de la tierra fue menos desigual y las distancias sociales más pequeñas que en otros países centroamericanos (Hall, 1982; Torres-Rivas, 1975). Según esta visión dominante, la debilidad relativa de la oligarquía y la fuerza de la clase media rural contribuyeron a crear un Estado más activo y gobiernos más sensibles a las demandas sociales desde el comienzo de la era republicana (Rueschemeyer, Huber y Stephens, 1992). Haciéndose eco de la literatura sobre desigualdad en América Latina (Banco Mundial, 2003; Engerman y Sokoloff, 1997) y de la literatura cada vez más influyente sobre instituciones y crecimiento (Acemoglu, Johnson y Robinson, 2005), estas explicaciones buscan la clave del éxito costarricense en el período colonial y en el desarrollo institucional posterior ${ }^{5}$.

Si bien es cierto que Costa Rica se benefició de una estructura económica menos desigual que la de otros países de América Latina, los datos empíricos nos hacen dudar de la validez de esta explicación histórica. Entre 1935 y 1937, la mortalidad infantil en Costa Rica fue de 159 niños por cada 1.000, más alta que en el Ecuador (145), El Salvador (137) y México (135) (Hytrek, 1999). Además, la distribución de la renta puede haber sido algo más equitativa que en los países vecinos, pero la desigualdad era, aun así, muy alta (Bowman y Baker, 2007). Dado que 40 años después Costa Rica tenía uno de los índices de mortalidad más bajos y una de las expectativas de vida más altas del mundo, cabe esperar que durante la segunda mitad del siglo XX ocurrieran cambios significativos que, desde luego, no se pueden explicar solo por la herencia colonial.

En explicaciones alternativas del éxito de Costa Rica se destaca la calidad de las instituciones estatales. La evidencia disponible con respecto al mejor desempeño relativo del servicio civil costarricense en comparación con el de los países vecinos parecería apoyar la tesis de las capacidades burocráticas: a fines de los años noventa, Costa Rica obtuvo el mayor puntaje latinoamericano y ocupó el décimo lugar entre los 35 países considerados por Evans y Rauch (1999) para evaluar la calidad burocrática. Sin embargo, la construcción de capacidades estatales no precedió sino que se produjo al mismo tiempo que se introducían las políticas que permitieron la doble incorporación. Así, las reformas constitucionales de 1946 y 1949 al servicio civil y la Ley de Administración Pública de 1953 se aprobaron paralelamente a los cambios que permitieron fortalecer el empleo y desarrollar la política social. Por otro lado, la capacidad estatal de por sí no permite explicar la doble incorporación en una perspectiva comparada: no en vano países con instituciones fuertes, como la provincia china de Taiwán y la República de Corea, contaron con instituciones sólidas sin tener necesariamente niveles altos de incorporación social.

Para Sandbrook y otros (2007), el éxito costarricense fue el resultado de la existencia de un Estado desarrollista, término acuñado para referirse a Estados capaces de disciplinar al sector privado y de hacer que este cumpla con ciertos criterios de desempeño (Amsden, 2001; Evans, 1995). Esta definición del Estado desarrollista no se corresponde con el Estado costarricense: si bien este logró expandir el empleo formal, fracasó en la creación de nuevas ventajas comparativas más dinámicas y de un sector industrial competitivo. Fue un Estado social en expansión antes que un Estado desarrollista capaz de conducir a la economía por una senda de innovación y aprendizaje tecnológico.

5 Esta explicación predominó en las entrevistas que realizamos en Costa Rica en julio y agosto de 2011. Véase también Bodenheimer (1970). 
De las explicaciones disponibles, la más influyente es la relativa al papel de la democracia en la promoción del desarrollo humano. La idea más extendida es que después de la guerra civil de 1948 las instituciones democráticas dieron lugar a la expansión de un partido socialdemócrata que fortaleció la burocracia y expandió la política social (Huber, 2005; Garnier y otros, 1991; Itzigsohn, 2000). Un segundo argumento subraya la naturaleza de las instituciones políticas (Gutiérrez Saxe y Straface, 2008; Lehoucq, 2010; Wilson, 1998). Concretamente, la aprobación de nuevas reglas electorales permitió que los actores políticos confiaran en las elecciones, que así se convirtieron en la única vía de acceso al poder (Lehoucq, 1998; Wilson, 1998). El sistema bipartidista y la expectativa de alternancia permitieron consolidar políticas dirigidas al "votante medio", ubicado en el centro del espectro político (Straface, 2008). A su vez, el hecho de que buena parte de la política pública se confiara a entes autónomos redujo la influencia de la competencia electoral y partidaria en sectores clave de la política pública y aseguró la continuidad en las políticas (Lehoucq, 2010; Straface, 2008). La combinación de autonomía institucional y alternancia partidaria habría fortalecido la política pública.

No cabe duda de que las dinámicas institucionales son importantes, en particular para dar cuenta de los legados históricos que han operado durante el período neoliberal más reciente, que no se estudia en detalle aquí pero sí en Martínez Franzoni y Sánchez-Ancochea (2013a). Sin embargo, otros países con democracias de larga duración, como Chile y el Uruguay antes de 1970 en América Latina y otros en distintas partes del mundo, tuvieron menos éxito en lo que respecta a asegurar la doble incorporación. En el caso de Costa Rica la explicación institucional es incompleta, por sí sola, para explicar la incorporación social y laboral registrada desde los años cincuenta. En primer lugar, porque las instituciones autónomas nunca fueron estrictamente autónomas: los partidos políticos buscaron influenciarlas de manera directa e indirecta. A comienzos de la década de 1950, por ejemplo, los actores sociales cercanos al PLN, como la Confederación de Trabajadores Rerum Novarum, tuvieron una participación activa en el nombramiento de directivos de los bancos y del Instituto Costarricense de Electricidad (Brenes, 1990). En segundo lugar, el PLN fue el único partido que participó en todas las elecciones presidenciales, el único con mayoría legislativa siempre (Hernández, 2009; Rovira, 1987) y el verdadero responsable de la creación de la mayoría de las instituciones autónomas. En tercer lugar, particularmente entre 1950 y 1980, la separación entre partidos políticos y burocracia estatal fue muy difusa. Muchas de las personas nombradas para ocupar altos cargos de las instituciones autónomas eran simpatizantes del PLN y apoyaban su proyecto político (Denton, 1969). En suma, la evidencia apunta a considerar al PLN y sus miembros como el actor clave detrás de la expansión del Estado, en general, y de la institucionalidad pública, en particular.

Sin duda, la democracia abrió espacios para el debate público y forzó a la élite política a responder, al menos parcialmente, a las demandas sociales. Sin embargo, el papel directo de la democracia formal en la expansión del Estado podría ser menos importante de lo que tiende a creerse y no fue condición suficiente, ni mucho menos, para el éxito. En primer lugar, porque muchos de los cambios más radicales en materia económica los realizaron los ganadores de la guerra civil de 1948 en el marco de un gobierno de facto, con poca legitimidad democrática per se ${ }^{6}$. En segundo lugar, en 1951 - año de su fundación - el PLN era una fuerza electoral pequeña, que venía de experimentar dos derrotas consecutivas en las elecciones de legisladores y para la Asamblea Constituyente ${ }^{7}$. EI PLN estaba entonces rodeado por un amplio conjunto de fuerzas de la oposición, desde la oligarquía cafetalera hasta los comunistas. En las elecciones de 1953, sin embargo, su candidato presidencial, José "Pepe" Figueres, obtuvo el 65\% de los votos (Hernández, 2009). En un cortísimo período, el flamante PLN combinó represión de los líderes de oposición, prohibición de la fuerza militar

6 El hecho de que desde su creación el PLN se presentara como el defensor de la democracia no cambia el hecho de que fueron sus líderes más connotados quienes impulsaron la guerra civil, eliminaron los poderes constitucionales, gobernaron bajo una Junta y demoraron 18 meses en entregar el poder al Presidente electo.

7 En la Asamblea Constituyente de 1949 los socialdemócratas obtuvieron 4 de 45 representantes (Rovira, 1987). Ese mismo año, en las elecciones ordinarias para renovar la mitad del Congreso obtuvieron 3 de 45 legisladores. 
prooligárquica, medidas específicas de política durante el período de gobierno de la Junta y promesas electorales para convertirse en la fuerza política dominante.

La idea de que Costa Rica fue una democracia plena durante la mayor parte de ese período es también cuestionable. Durante la década de 1950, oponentes partidarios y activistas sindicales fueron exiliados o encarcelados. Entre 1949 y 1975, el sistema político costarricense mantuvo exclusiones antidemocráticas. En el segundo párrafo del artículo 98 de la Constitución se establecía que la Asamblea Legislativa podía prohibir los partidos políticos que se considerasen antidemocráticos. Si bien la extensión y la intensidad de la exclusión disminuyeron hacia el final del período, dicha exclusión significó que un grupo de ciudadanos carecieron de la misma libertad de pensamiento y de organización o de las mismas opciones de votación que el resto de la población ${ }^{8}$.

En suma, entre 1950 y 1980, el proceso de consolidación democrática ocurrió de manera simultánea a las transformaciones económicas y sociales (Programa Estado de la Nación, 2001; Vargas-Cullell, Rosero-Bixby y Seligson, 2005). En general, la competencia electoral simultáneamente promovió e inhibió cambios en la política social, codo a codo con otros factores como la iniciativa burocrática y el papel de las organizaciones internacionales (McGuire, 2010).

\section{La élite emergente y la doble incorporación}

Argumentamos que la doble incorporación se apoyó en una expansión acelerada de la presencia estatal en la economía y en la provisión social, que fue, a su vez, resultado de los intereses de una élite emergente formada por distintos segmentos de la clase media. Agrupada alrededor del PLN, esa élite utilizó al Estado en su propio beneficio, concretamente para: i) apoyar las actividades económicas en que tenía intereses; ii) ampliar su base de apoyo social mediante la expansión del empleo público, y iii) manejar y suprimir el conflicto.

A la vez, muchas de las políticas promovidas por el PLN solo pueden entenderse en el contexto de ideas que estaban disponibles internacionalmente. Los políticos, y en particular los actores tecnopolíticos, tomaron diferentes ideas selectivamente y las adaptaron a las condiciones nacionales y a sus propios objetivos políticos. Antes de la década de 1980, esas políticas se centraron en la importancia de la sustitución de importaciones, en la seguridad social universal y, más tarde, en la asistencia social.

Seguidamente describimos cómo esa élite emergente llegó al poder, cómo el proceso de construcción del Estado en favor de grupos específicos contribuyó a la incorporación social y laboral, y cómo las ideas internacionales dieron forma a las políticas.

\section{La nueva élite y la expansión del Estado entre 1950 y 1970}

El principal objetivo del PLN desde sus inicios fue la creación de oportunidades económicas para pequeños y medianos propietarios y profesionales urbanos (Bodenheimer, 1970). Esto requería que el partido se convirtiera en una fuerza electoral dominante, algo que Figueres logró con su aplastante victoria de 1953. Una vez en el gobierno, Figueres expandió el empleo público (que entre 1950 y 1958 pasó del $6 \%$ al 10\% de la fuerza laboral activa), introdujo el décimo tercer salario anual entre

8 Rovira (1990) sitúa a la consolidación democrática en 1958, cuando se produjo la alternancia partidaria. En este artículo la ubicamos en 1970, cuando se legalizó el Partido Comunista. 
los funcionarios públicos (como un mecanismo explícito para distribuir el superávit presupuestario) y promovió sindicatos proliberacionistas ${ }^{9}$.

Debido a que pequeños y medianos propietarios como Figueres tenían una enorme necesidad de financiamiento, el PLN también expandió el crédito y promovió la modernización del sector agrícola. A fines de los años cincuenta, más de un tercio de los créditos nacionales se canalizaban al sector agrícola y la producción de granos básicos como frijol, arroz y maíz era promovida activamente desde el Consejo Nacional de Producción (Brenes, 1990). Las crecientes intervenciones beneficiaron directamente al grupo cercano a Figueres. Varios de los líderes del PLN tenían intereses crecientes en ranchos de ganado y recibían crédito barato en cantidades abundantes (Aguilar y Solís, 1988). El propio Figueres y su hermano eran dueños de una de las fincas cafetaleras más grandes del país, localizada en Turrialba (Winson, 1989). Si bien la élite tradicional también se benefició del apoyo estatal a la modernización de la producción de café, el Estado le impuso más demandas. Por ejemplo, en 1954 se introdujo un nuevo impuesto debido al cual "las 90 empresas más grandes del país verían sus impuestos incrementarse de 5 millones de colones al año a 14,5 millones de colones al año" (Bowman y Baker, 2007, pág. 38).

Con la aprobación de la Ley de Protección y Desarrollo Industrial en 1959 y la incorporación de Costa Rica al Mercado Común Centroamericano (MCCA) en 1963, el centro de la política económica se desplazó de la agricultura a la promoción industrial. Ambas medidas fueron promovidas por la élite del PLN, que vio en ellas una oportunidad de expandir sus bases económicas ${ }^{10}$. La estrategia económica enfrentó una oposición feroz de la oligarquía tradicional agroexportadora. Dichos grupos, que estaban en el gobierno a principios de la década de 1960, rehusaron firmar el acuerdo constitutivo del MCCA, por lo que la incorporación de Costa Rica recién tuvo lugar luego de la victoria electoral del PLN en 1962.

Tanto los subsidios al préstamo como la protección del mercado interno generaron altas rentas empresariales, definidas estas como oportunidades de ingreso por encima de los niveles que se obtendrían en una situación de libre mercado (Akyuz y Gore, 1996). González-Vega, por ejemplo, estima que en 1974 el acceso preferencial al crédito barato se tradujo en "un subsidio implícito del 30\% al año” (1990, pág. 21). También demuestra que en la agricultura la transferencia implícita de los bancos públicos fue equivalente a alrededor de un quinto del valor agregado total. Aunque la mayoría de los datos disponibles sobre el nivel de protección se refieren a fines de la década de 1970 y a inicios de la década de 1980, los aranceles ya eran igualmente altos durante los años sesenta. En 1980, la tasa efectiva de protección para el sector industrial en su conjunto fue del 139\%, con una varianza de entre el $45 \%$ y el $388 \%$ dependiendo de la actividad. Los productos textiles y de cuero, así como los muebles y los productos de madera, recibieron una protección particularmente generosa (Salazar, 1990). De acuerdo con cálculos de Monge González y González-Vega (1995, pág. 134), el resultado de las transferencias de consumidores a productores fue equivalente a un $16 \%$ del producto interno bruto (PIB) en 1996, y probablemente haya sido todavía más alto en décadas anteriores ${ }^{11}$.

Por supuesto, durante ese período muchos otros gobiernos promovieron la creación de rentas que contribuyeron a producir altos beneficios empresariales (Amsden, 2001). Como muchos de esos

9 Entre 1950 y 1980, el sistema político costarricense tuvo dos bloques: el PLN y grupos conservadores que en ocasiones lograron construir coaliciones anti-PLN exitosas. Entre 1951 y 1978, los conservadores ganaron dos elecciones presidenciales pero nunca obtuvieron la mayoría parlamentaria. Antes de 1970, dado que el Partido Comunista era ilegal, el PLN no enfrentó una amenaza electoral desde la izquierda.

10 Gracias a estas disposiciones y a otras medidas de protección, la Cámara de Industrias, conformada por empresarios dependientes de la subvención pública, se convirtió en una partidaria entusiasta del PLN. Una encuesta realizada a finales de la década de 1970 mostraba que más de dos tercios de los miembros de la Junta Directiva de la Cámara eran partidarios del PLN (Vega, 1982).

${ }^{11}$ Aunque es cierto que el cálculo de estas transferencias se basa en supuestos económicos muy discutibles (véase Ocampo y Taylor, 1998), da una idea de la magnitud de los subsidios que recibían muchos productores. 
gobiernos, el de Costa Rica tuvo poco éxito en lo que respecta a lograr que esas rentas se tradujeran en nuevas ventajas comparativas y en un crecimiento sistemático de la productividad del sector manufacturero (Brenes, 1990; Lizano, 1999). Lo que sí fue particular y exitoso en Costa Rica fue el modo en que se distribuyeron dichas rentas. Aunque buena parte de la inversión pública se dirigió a una pequeña parte de la nueva élite, pequeños y medianos productores de todo el país también se vieron beneficiados. Por ejemplo, en 1952 el Banco Nacional de Costa Rica, a través de sus consejos rurales para el desarrollo económico, otorgó 20.000 préstamos que favorecieron a un cuarto de todos los productores agrícolas, y en 1976 se dieron más de 24.000 préstamos (González-Vega, 1990). Una buena parte de esos préstamos fueron además a instituciones públicas que, a su vez, se expandieron rápidamente y crearon una considerable cantidad de empleos en todo el país. El proteccionismo también se tradujo en la creación de nuevas empresas. De hecho, a fines de la década de 1970, el $40 \%$ de los empresarios industriales eran hijos de padres sin un negocio propio (Garnier e Hidalgo, 1991). En resumen, las políticas económicas promovidas por el PLN durante ese período ayudaron a fortalecer a la nueva élite y, al hacerlo, facilitaron la incorporación al mercado de una creciente clase media conformada por empleados públicos y por propietarios de pequeñas empresas en todos los sectores de la economía (Rodríguez, 1997).

En lo que respecta a la política social, hay que reconocer que durante los años cincuenta y sesenta esta fue menos prioritaria para el PLN que la modernización económica. De hecho, "la mayoría de la infraestructura del bienestar social se había creado antes de la revolución de 1948" y "no hubo innovaciones radicales" en ese período (Bodenheimer, 1970, pág. 71; véase también Winson, 1989). Aun así, el gasto social y la cobertura de los servicios aumentaron gradualmente debido a tres factores distintos. Primero, el PLN usó los programas sociales como un instrumento para expandir sus bases de apoyo. Esto es evidente, por ejemplo, cuando se considera la aprobación de la ley que estableció la universalización de la seguridad social en 1961. De acuerdo con el principal impulsor de la ley, el diputado Enrique Obregón Valverde, con ella el PLN -entonces en la oposición pero con mayoría legislativa - quería aparecer como responsable de la consolidación de la seguridad social y así compensar el hecho de que la institución hubiera sido creada por los socialcristianos en la década de $1940^{12}$. Segundo, la burocracia a cargo de las políticas de pensiones y de salud exigió la creación de nuevos mecanismos de financiación para asegurar la expansión y la sostenibilidad de la seguridad social (Rosemberg, 1983). Tercero, las pocas iniciativas sociales nuevas que el PLN promovió durante ese período, aunque estaban orientadas a fomentar la transformación económica, también contribuyeron a la incorporación social. Este es el caso de programas de vivienda y, todavía más claramente, del Instituto Nacional de Aprendizaje (INA).

La creación del INA en 1965 tuvo por objeto apoyar el proceso de industrialización a través del fomento de la capacitación de la clase trabajadora. La Ley 3.506 que dispuso su creación fue propuesta por el Ministro de Trabajo Alfonso Carro Zúñiga, con el asesoramiento de la Organización Internacional del Trabajo (OIT) y de expertos israelíes (Congreso Constitucional, 1965). El PLN quería incrementar las capacidades productivas de la fuerza laboral, particularmente en el sector manufacturero, y ampliar las oportunidades de empleo para la población joven o de escasos ingresos. Al mismo tiempo, el INA debía contribuir a aumentar la ganancia empresarial y, así, incrementar también la base social del partido entre los sectores económicos más acomodados. La Ley fue apoyada de manera entusiasta por la Cámara de Industrias, los medios de comunicación y el Congreso.

La expansión del empleo público fue otro instrumento fundamental para aumentar las bases de apoyo del PLN. Mientras que en 1960 el empleo público representaba menos del 10\% de la población económicamente activa, 20 años después había aumentado hasta alcanzar cerca del 18\%. En términos absolutos, se pasó de 30.000 a 150.000 puestos de trabajo en el sector público

12 Entrevista a Obregón Valverde, realizada el 10 de agosto de 2011. 
(CLAD, 2007). Desafortunadamente, el PLN utilizó también otros métodos menos democráticos para reducir los conflictos y minimizar la oposición al proyecto modernizador, entre los que cabe destacar la cooptación y la represión de los sindicatos. Debido a la ausencia de un fuero sindical que protegiera a la dirigencia contra despidos antisindicales, los sindicatos del sector privado fueron sistemáticamente reprimidos (Castro Méndez y Martínez Franzoni, 2010). Hacia mediados de la década de 1970, solo el $5 \%$ de los empleados privados eran miembros de sindicatos. En el sector público se produjo una combinación de represión con cooptación. En 1971, el Ministro de Trabajo reconoció que no existían "las libertades sindicales como tales" (Aguilar, 1989, pág. 174). Cuando se estableció el derecho a huelga los requerimientos fueron tan altos (por ejemplo, las huelgas debían ser apoyadas por el $60 \%$ de los trabajadores involucrados) que su práctica se vio severamente limitada, y virtualmente todas las huelgas resultaron ilegales (Castro Méndez y Martínez Franzoni, 2010). De las 182 huelgas contabilizadas entre 1972 y 1983, y de las 159 declaradas entre 1990 y 1998, solo 5 se consideraron legales (Donato y Rojas Bolaños, 1987; Programa Estado de la Nación, 2001). Ya algo más tarde, en 1984, y con el apoyo de las cámaras empresarias, el gobierno sancionó la Ley de Asociaciones Solidaristas que creó organizaciones mutuales de ahorro y crédito integradas por trabajadores y trabajadoras, con participación de los empleadores. Si bien formalmente estas organizaciones solidaristas eran complementarias a las sindicales, en la práctica se convirtieron en un poderoso instrumento de política antisindical porque recurrentemente se buscó atribuirles funciones de representación de intereses laborales de trabajadores y trabajadoras (Castro Méndez, 2014).

\section{El renovado impulso estatal durante los años setenta}

El PLN, en el poder entre 1970 y 1978, buscó enfrentar la crisis económica internacional de comienzos de los años setenta y las crecientes limitaciones de la producción manufacturera dándole un nuevo papel al sector público. Expandir la función económica del Estado se volvió más importante que la promoción de oportunidades para la acumulación privada. La creación de la Corporación Costarricense de Desarrollo (CODESA) en 1972 fue el primer paso de esa nueva estrategia para la incorporación laboral. Inicialmente se esperaba que la CODESA fomentara la inversión en nuevos sectores de la economía y estimulara alianzas público-privadas. Su junta directiva estaba integrada por representantes de asociaciones empresariales, incluida la Cámara de Industrias, y en sus comienzos no compitió con el sector privado ${ }^{13}$. En 1975, sin embargo, la CODESA se había expandido a un número creciente de sectores, incluidos los del azúcar, el algodón y el cemento, y también en el mercado de valores. Había recibido además un amplio apoyo crediticio: en 1983, los préstamos del Banco Central de Costa Rica a la CODESA representaron la mitad de todos los préstamos al sector privado y el $18 \%$ del crédito doméstico total.

Con la creación de la CODESA y la profundización del papel del Estado, el PLN perseguía un doble objetivo. Por un lado, se trataba de crear una "burocracia burguesa" formada por miembros del partido que iban aumentando su poder y se iban enriqueciendo (Sojo, 1984). Por otro lado, se lograba expandir el empleo público en un momento en que el mercado laboral experimentaba una situación particularmente difícil. No debe resultar sorprendente entonces que casi desde el principio el sector privado se opusiera a la expansión de la CODESA y se volviera crecientemente crítico del PLN (Vega, 1980).

En el caso de la política social, durante la década de 1970 la élite del PLN apostó por una profundización del papel del Estado y, a la vez, por darle un giro al enfoque. Hasta entonces, las acciones para incrementar la incorporación social y laboral se habían centrado en el Valle Central y habían beneficiado principalmente a la clase media. Entre 1963 y 1973, por ejemplo, el ingreso

\footnotetext{
${ }^{13}$ Entrevista al asesor técnico-político que asistía al Presidente Oduber, realizada en agosto de 2011.
} 
recibido por las personas ubicadas en el medio de la estructura social (deciles 4 a 8) se incrementó del $30 \%$ al $40 \%$. Sin embargo, la participación del 20\% de la población con menores ingresos disminuyó ligeramente, al pasar del $6 \%$ al 5,4\% (OFIPLAN, 1982) ${ }^{14}$. Los intentos por redistribuir la tierra durante la década de 1960 fracasaron y los campesinos sin tierra que habitaban fuera del Valle Central siguieron excluidos del proceso de modernización (Seligson, 1980) ${ }^{15}$.

El PLN se enfrentó a un creciente conflicto social a principios de los años setenta: entre 1971 y 1974, 2.240 familias ocuparon ilegalmente más de 91.000 hectáreas (Cortés y León, 2008). Gran parte de la protesta fue canalizada por movimientos progresistas con vínculos con el todavía ilegal Partido Comunista y con otros partidos de izquierda cuya presencia en zonas rurales se incrementó considerablemente ${ }^{16}$. Para hacer frente al conflicto el PLN introdujo nuevos programas sociales orientados a los pobres, sobre todo de las zonas rurales. En 1970, Figueres creó el Instituto Mixto de Ayuda Social (IMAS) para transferir subsidios a personas que vivían en condiciones de pobreza extrema. En 1974, más significativa aún fue la creación del Fondo de Desarrollo Social y Asignaciones Familiares (FODESAF) bajo el gobierno de Oduber. Durante el primer año de funcionamiento, el FODESAF canalizó el 1,4\% del PIB anual (Trejos citado en Rovira, 1987). En cuanto fondo, se trató de una innovación en América Latina, enfocado en financiar servicios dirigidos a las personas hasta entonces excluidas que se canalizaron mediante servicios públicos e instituciones públicas ya existentes que atendían a la población no pobre. El FODESAF también creó nuevos programas, entre los que se incluyeron pensiones no contributivas, atención primaria de la salud y comedores escolares, que se implementaron desde instituciones de carácter universal ${ }^{17}$.

\section{El papel de las ideas internacionales entre 1950 y 1980}

Si bien los intereses de la élite del PLN nos ayudan a entender la dirección general de la política pública, no son condición suficiente para explicar sus rasgos concretos. Para ello se requiere abordar el papel y el origen de las ideas y analizar cómo estas fueron cambiando a lo largo del tiempo. Concretamente, argumentamos que los actores internacionales y las ideas que esos actores promovieron fueron extremadamente importantes para la formación de la política pública.

A modo de ejemplo, las reformas sociales y laborales implementadas durante la década de 1940 se promovieron en un entorno muy influenciado por el reformismo anticomunista del New Deal impulsado por el Presidente Roosevelt en los Estados Unidos (Acuña, 1995). También, el seguro de salud creado en 1941 fue marcadamente influido por recomendaciones internacionales que sugerían evitar regímenes estratificados por ocupaciones y más bien promover sistemas unificados para todas las personas (Martínez Franzoni y Sánchez-Ancochea, 2012; Seekings, 2010).

Durante las tres décadas siguientes, paradigmas regionales e internacionales moldearon diversas políticas económicas y sociales. Contrariamente a lo ocurrido en los países pioneros de América del Sur en que el cambio en la estructura productiva precedió a las políticas explícitas de sustitución de importaciones (Thorp, 1998), en Centroamérica las políticas proteccionistas e industriales respondieron a ideas internacionales cuyo objeto era transformar la estructura económica, y no viceversa. Por medio del MCCA, la CEPAL y el Gobierno de los Estados Unidos desempeñaron

\footnotetext{
${ }^{14}$ Los datos se basan en el ingreso mensual nominal de los hogares y deben ser interpretados con cautela en cuanto se desconoce la confiabilidad de las encuestas llevadas a cabo en esa época.

15 Hacia mediados de los años setenta, la nueva institución encargada de la distribución de la tierra había transferido solo el 3\% de la tierra arable y había beneficiado al 1,7\% de las familias campesinas sin tierra (Seligson, 1984).

${ }^{16}$ Las protestas sociales contra proyectos de inversión extranjera también se intensificaron en el sector urbano. El caso más emblemático fue el de ALCOA.

17 Entrevista a Kyra del Castillo, participante en el diseño y el impulso inicial del FODESAF, realizada en agosto de 2011.
} 
un papel fundamental en la implementación de la sustitución de importaciones. Algo similar pasó en materia de seguridad social, que se creó en ausencia de las presiones de la clase trabajadora que le habían dado origen en el Cono Sur. En su lugar, la experiencia de otros países, en particular Chile, se combinó con las prescripciones políticas de la OIT para promover que países rezagados como Costa Rica se pusieran al día con los modelos de incorporación social considerados deseables en ese momento.

En la década de 1950, la CEPAL promovió la industrialización mediante un sistema llamado de "integración limitada con reciprocidad" (Bulmer-Thomas, 1987). Concretamente, propuso crear monopolios regionales regulados por el Estado para distribuir nuevas industrias de manera equitativa entre los países miembros. Detrás del Tratado Multilateral de Libre Comercio e Integración Económica Centroamericana estuvo el modelo de la CEPAL que excluía del libre mercado numerosos productos agrícolas como granos básicos, algodón, café y azúcar. En la integración económica centroamericana también se reflejaron las ideas del Gobierno de los Estados Unidos. Concretamente, la administración de Eisenhower favoreció el libre comercio entre todos los países miembros e impulsó la idea de planificar monopolios regionales. Estas ideas estuvieron detrás del Tratado Tripartito de febrero de 1960 que se convirtió en la base del Tratado General de Integración Económica Centroamericana aprobado en diciembre de ese año por El Salvador, Guatemala, Honduras y Nicaragua, al cual tres años después se incorporó Costa Rica.

Las ideas internacionales relativas a la sustitución de importaciones tuvieron repercusiones marcadamente diferentes en la realidad de cada país centroamericano. Costa Rica fue más exitosa que el resto al menos por dos razones. En primer lugar, la protección industrial contribuyó a una expansión gradual de la política social basada en los impuestos a la planilla. La mayoría de las empresas podían despreocuparse de los altos costos laborales resultantes porque no competían internacionalmente (Lizano, 1999). La situación de los agroexportadores era más preocupante, pero muchos de ellos (particularmente aquellos que contrataban trabajo temporal, incluidos los productores de café) estaban exentos del mencionado impuesto. A diferencia de Asia Oriental, donde las exportaciones de manufacturas intensivas en mano de obra hicieron que los gobiernos y las empresas opusieran resistencia a los regímenes de seguridad social que aumentarían los costos de mano de obra (Haggard y Kaufman, 2008, pág. 9), en Costa Rica la industrialización mediante sustitución de importaciones facilitó la expansión del gasto público. En segundo lugar, la política industrializadora no solo benefició a las grandes compañías sino también a las pequeñas y medianas empresas y cooperativas, algo excepcional en comparación con el resto de América Latina.

Las ideas internacionales también desempeñaron un papel importante en la formación de los programas de asistencia social a mediados de los años setenta. Siguiendo el ejemplo de Francia, el Uruguay y otros países, así como recomendaciones de política por ese entonces bien establecidas por la OIT, Figueres y su Ministro de Trabajo, Jiménez Veiga, propusieron la creación de las asignaciones familiares, es decir, un ingreso transferido por cada hijo o hija a los trabajadores formales de ingresos bajos. Este programa, sin embargo, llegó en un momento de creciente influencia de las ideas de transformación social que siguieron a la Revolución Cubana de 1959, que subrayaban la necesidad de ir más allá de las transferencias monetarias, mediante la inclusión de servicios bajo una respuesta integrada y multidimensional a la pobreza. Esta visión se reflejó en la Alianza para el Progreso de Kennedy, en la guerra contra la pobreza de Johnson, en las nuevas intervenciones basadas en el enfoque de las necesidades básicas promovido por el Banco Mundial y en las ideas de la CEPAL y de la Internacional Socialista. El Presidente Oduber (1974-1978) y su Vicepresidente Castillo estaban más en sintonía con estas ideas que Figueres. Oduber había sido Vicepresidente de la Internacional Socialista y Castillo había trabajado para la CEPAL y para el Sistema de la Integración Centroamericana. Ganadas las elecciones en febrero de 1974, ambos buscaron, con éxito, transformar el proyecto original para convertirlo en un fondo de desarrollo social. 


\section{Los vaivenes de la doble incorporación}

La incorporación puede reducirse, así como la exclusión puede aumentar, si se promueven políticas inadecuadas en momentos de crisis. En las últimas décadas, Costa Rica ha enfrentado dificultades para mantener la doble incorporación alcanzada en las décadas anteriores. En parte como resultado del nuevo modelo abierto orientado a las exportaciones, la fuerza laboral costarricense se ha bifurcado cada vez más entre aquellas personas con trabajos formales bien remunerados en sectores dinámicos de la economía, como el turismo, las finanzas y el ensamblaje de alta tecnología, y aquellas que solo tienen acceso a empleos informales no calificados y de baja productividad en otras áreas, principalmente volcadas al mercado interno. En 2006, el sector informal representó el 35\% de la población trabajadora en comparación con el 20\% a comienzos de la década de 1980. Entre 1984 y 2009, el salario mínimo medio en términos reales creció a una tasa media anual de apenas el 0,7\% y la desigualdad salarial se incrementó considerablemente (Programa Estado de la Nación, 2011). En términos de incorporación social se ha mantenido una cobertura casi universal en materia de salud y una cobertura muy alta en materia de pensiones, así como elevadas tasas de matrícula en educación primaria y secundaria, aunque con crecientes problemas de calidad y enormes presiones para privatizar la prestación de los servicios (Martínez Franzoni y Sánchez-Ancochea, 2013b).

Buena parte de los problemas se deben, precisamente, al debilitamiento de la contribución de las dos variables independientes aquí presentadas al proceso de incorporación laboral y social: la composición y los intereses de la élite, y las ideas internacionales. En primer término, la élite costarricense responsable del éxito anterior se ha dividido y una gran parte de ella depende cada vez menos de la expansión del mercado interno. Las divisiones dentro del PLN ocasionadas por diferencias sobre el modelo de desarrollo óptimo ya habían surgido en la segunda mitad de la década de 1970, pero se intensificaron a comienzos de los años ochenta (Lizano, 1999). Nuevos líderes como Oscar Arias, Presidente de la República en los períodos 1986-1990 y 2006-2010; Eduardo Lizano, Presidente del Banco Central de Costa Rica en varias ocasiones, y otros promovieron un cambio cuantitativo y cualitativo de la participación del Estado en asuntos económicos. Las tensiones giraron en torno a la conveniencia o no de tener empresas públicas directamente involucradas en la producción y de mantener un sistema bancario exclusivamente público. Estos líderes representaban, en buena medida, los intereses de grupos empresariales reunidos en torno al PLN que habían ido creciendo y colaborando con las empresas transnacionales durante los años setenta y no tenían ya interés en apoyar el modelo económico costarricense tradicional.

Las divisiones dentro de las élites se han profundizado desde entonces y el poder de los grupos interesados en promover las exportaciones y las finanzas ha crecido de forma sostenida. La agenda del PLN - que se ha ido acercando cada vez más a la de la oposición socialcristiana (Rovira, 2004) - se ha centrado de forma creciente en crear mejores condiciones para los inversores extranjeros, expandir la vinculación entre estos y las empresas costarricenses, y apelar a una política social compensatoria. Fuera del PLN, los grupos empresariales emergentes están más interesados en fortalecer sus lazos con las empresas extranjeras y en expandirse en el mercado regional que en promover la capacidad de consumo de la población costarricense (Bull y Kasahara, 2013; SánchezAncochea, 2003; Segovia, 2005).

En segundo término, los cambios en las ideas internacionales también permiten explicar la dirección de las políticas de Costa Rica. A nivel mundial, el Consenso de Washington cuestionó las ideas socialdemócratas tradicionales y ofreció una justificación teórica para la liberalización comercial y la desregulación financiera. Simultáneamente, países como Singapur e Irlanda se convirtieron en ejemplos poderosos de cómo atraer a determinadas compañías extranjeras y proporcionar incentivos a sectores particulares de la economía en detrimento de otros (Mortimore y Vergara, 2004; Sánchez- 
Ancochea, 2009). Siguiendo estas experiencias, el PLN apoyó de manera decidida la promoción de la inversión extranjera en alta tecnología, una política que incrementó la competitividad, pero que hizo relativamente poco para promover la incorporación laboral a nivel agregado.

\section{Implicaciones}

A pesar de los cambios experimentados durante las últimas décadas, el caso costarricense ofrece lecciones importantes, tanto en términos teóricos como en materia de políticas. A nivel teórico, muestra la necesidad de considerar seriamente la composición de la élite y los incentivos a partir de los cuales esta asume el proceso de construcción del Estado. El análisis que aquí presentamos permite observar que el problema para la incorporación no es que la élite busque controlar el Estado en su propio beneficio: puede ocurrir que estos grupos busquen su propio beneficio y, aun así, contribuyan a alcanzar resultados positivos en términos de doble incorporación, algo que autores como Acemoglu y Robinson (2012) no parecen tener en cuenta. Por ejemplo, la promoción del empleo público, de empresas pequeñas y medianas y de cooperativas antes de la década de 1980 en Costa Rica refleja, precisamente, la capacidad de varios segmentos de la clase media de construir un Estado a la medida de sus propios intereses.

De igual manera, el estudio aquí presentado permite observar que los incentivos que la élite tiene para promover la transformación laboral y la incorporación social no necesariamente van de la mano: no hay razón para esperar que un Estado centrado en el cambio estructural sea producto del mismo proceso que da lugar a políticas sociales universales, o viceversa. Lo que hace particularmente interesante al caso de Costa Rica es la convergencia entre una transformación productiva generadora de buenos empleos y una política social expansiva.

La principal implicación de esta investigación para la construcción de política pública es la insistencia en la importancia de entender quiénes conforman las élites y qué buscan - en particular del Estado - y de situar las innovaciones en el marco de ambientes internacionales de política pública. El acento en las élites nos permite tender un cable a tierra en términos de las posibilidades y limitaciones estructurales que existen para la doble incorporación (véase, en el mismo sentido, Schneider, 2012). Mientras tanto, las ideas internacionales desempeñan un papel central en la legitimación de las reformas y el mapeo del rango de opciones posibles en diferentes momentos, e influyen considerablemente en las características específicas de los programas que le dan forma al Estado.

Si el análisis presentado en este artículo es correcto, entonces el énfasis que desde la década de 2000 existe en la región, tanto en la política industrial (UNRISD, 2010) como en las políticas sociales universales (Mkandawire, 2006; Filgueira y otros, 2006), representa una oportunidad valiosa para que países con trayectorias de escasa incorporación social y laboral promuevan cambios en la política pública. A la vez, para que los países pongan al Estado al servicio de la doble incorporación, estas ideas requieren de actores que las compartan, las adapten y las expresen en decisiones de política pública. Así, es preciso saber hasta qué punto los países cuentan con élites emergentes que estén dispuestas a poner en práctica esta nueva senda de desarrollo.

\section{Bibliografía}

Acemoglu, D., S. Johnson y J. Robinson (2005), "Institutions as fundamental cause of long-run growth", Handbook of Economic Growth, Ph. Aghion y S. Durlauf (eds.), Amsterdam, Elsevier.

Acemoglu, D. y J. Robinson (2012), Why Nations Fail: The Origins of Power, Prosperity and Power, Nueva York, Crown Business. 
Acuña, M. (1995), Clays, Ivonne: testigo de la neurosis de una administración, San José.

Aguilar, M. (1989), Clase trabajadora y organización sindical en Costa Rica, 1943-1971, San José, Instituto Costarricense de Estudios Sociales (ICES)/Editorial Porvenir.

Aguilar, I. y M. Solís (1988), La élite ganadera en Costa Rica, San José, Editorial de la Universidad de Costa Rica.

Akyuz, Y. y Ch. Gore (1996), "The investment-profits nexus in East Asian industrialization”, World Development, vol. 24, N³, Amsterdam, Elsevier.

Amsden, A. (2001), The Rise of the Rest. Challenges to the West from Late- Industrializing Economies, Nueva York, Oxford University Press.

Amsden, A., A. di Caprio y J. Robinson (2012), The Role of Elites in Economic Development, Oxford, Oxford University Press.

Banco Mundial (2003), Inequality and Poverty in Latin America: Breaking with History?, Washington, D.C., World Bank Group.

Banerjee, A. y E. Duflo (2011), Poor Economics: A Radical Rethink of the Way to Fight Poverty, Nueva York, Public Affairs.

Bodenheimer, S. (1970), "The social democratic ideology in Latin America: the case of Costa Rica's Partido Liberación Nacional”, Caribbean Studies, vol. 10, Nº 3.

Bowman, K. y S. Baker (2007), "Noisy regimes, causal processes, and democratic consolidation: the case of Costa Rica", The Latin Americanist, vol. 50, № 2.

Brenes, L. (1990), La nacionalización bancaria en Costa Rica. Un juicio histórico, San José, Facultad Latinoamericana de Ciencias Sociales (FLACSO).

Bull, B. e Y. Kasahara (2013), "The transformation of Central American economic elites: from local tycoons to transnational business groups", Handbook of Central American Governance, D. Sánchez-Ancochea y S. Martí i Puig (eds.), Londres, Routledge.

Bulmer-Thomas, V. (1987), The Political Economy of Central America since 1920, Cambridge, Cambridge University Press.

Castro Méndez, M. (2014), "Arreglos directos y comités permanentes de trabajadores desde la perspectiva de la libertad sindical en Costa Rica”, Revista de la Sala Segunda de la Corte Suprema de Justicia de Costa Rica, №11, San José.

Castro Méndez, M. y J. Martínez Franzoni (2010), "Un modelo exitoso en la encrucijada: límites del desencuentro entre régimen laboral y de bienestar en Costa Rica", Revista Centroamericana de Ciencias Sociales, vol. $7, \mathrm{~N}^{\circ} 1$.

CEPAL (Comisión Económica para América Latina y el Caribe) (2014), Pactos para la igualdad: hacia un futuro sostenible (LC/G.2586(SES.35/3)), Santiago.

CLAD (Centro Latinoamericano de Administración para el Desarrollo) (2007), "Evolución del empleo en el sector público de Costa Rica (1950-1987)" [en línea] www.clad.org.ve/siare/tamano/deca1980/80cua51.hml.

Collier, R. y D. Collier (1991), Shaping the Political Arena: The Incorporation of the Labor Movement in Latin America, Princeton, Princeton University Press.

Congreso Constitucional (1965), "Ley de Creación del Instituto Nacional de Aprendizaje: Ley No. 3506”, mayo.

Cortés, A. y A. León (2008), "Costa Rica: conflictividad social y distribución, 1950-2005”, Research Report, Policy Regime and Poverty Reduction, Costa Rican Case, Ginebra, Instituto de Investigaciones de las Naciones Unidas para el Desarrollo Social (UNRISD).

Denton, Ch. (1969), "Bureaucracy in an immobilist society: the case of Costa Rica", Administrative Science Quarterly, vol. 14, N 3, Sage Publications.

Donato, E. y M. Rojas Bolaños (1987), Sindicatos y política económica, 1972-1986, San José, Alma Mater.

Engerman, S. y K. Sokoloff (1997), "Factor endowments, institutions, and differential paths of growth among new world economies: a view from economic historians of the United States", How Latin America Fell Behind, S. Haber (ed.), Stanford, Stanford University Press.

Evans, P. (1995), Embedded Autonomy: States and Industrial Transformation, Princeton, Princeton University Press.

Evans, P. y J. Rauch (1999), "Bureaucracy and growth: a cross-national analysis of the effects of 'Weberian' state structures on economic growth", American Sociological Review, vol. 64, № 5, American Sociological Association.

Filgueira, F. (2005), "Welfare and democracy in Latin America: the development, crises and aftermath of universal, dual and exclusionary welfare states", Documento de Trabajo, Ginebra, Instituto de Investigaciones de las Naciones Unidas para el Desarrollo Social (UNRISD). 
Filgueira, F. y otros (2006), "Universalismo básico: una alternativa posible y necesaria para mejorar las condiciones de vida", Universalismo básico. Una nueva política social para América Latina, C.G. Molina (ed.), Washington, D.C., Banco Interamericano de Desarrollo (BID).

Garnier, L. y R. Hidalgo (1991), "El Estado necesario y la política de desarrollo", Costa Rica: entre la ilusión y la desesperanza. Una alternativa para el desarrollo, L. Garnier y otros (eds.), San José, Ediciones Guayacán.

Garnier, L. y otros (1991), Costa Rica: entre la ilusión y la desesperanza. Una alternativa para el desarrollo, San José, Ediciones Guayacán.

González-Vega, C. (1990), "Política de intermediación financiera en Costa Rica: cuatro décadas de evolución", Políticas económicas en Costa Rica, vol. 2, C. González-Vega y E. Camacho Mejía (eds.), San José, Academia de Centroamérica.

Goodman, R. el. Peg (1996), "The East Asian welfare state: peripatetic learning, adaptive change and nationbuilding", Welfare States in Transition. National Adaptations in Global Economies, G. Esping-Andersen (ed.), Londres, Sage Publications.

Gutiérrez Saxe, M. y F. Straface (2008), "Antecedentes: política y economía en la Costa Rica de los últimos 50 años", Democracia estable ¿alcanza? Análisis de la gobernabilidad en Costa Rica, M. Gutiérrez Saxe y F. Straface (eds.), Washington, D.C., Banco Interamericano de Desarrollo (BID).

Haggard, S. y R. Kaufman (2008), Development, Democracy, and Welfare States: Latin America, East Asia, and Eastern Europe, New Jersey, Princeton University Press.

Hall, C. (1982), El café y el desarrollo histórico-geográfico de Costa Rica, San José, Editorial de Costa Rica.

Hernández, G. (2009), Reseña de las elecciones de 1974, San José, Instituto de Investigaciones Sociales (IIS)-Universidad de Costa Rica (UCR).

Huber, E. (2005), "Inequality and the State in Latin America" [en línea] http://citeseerx.ist.psu.edu/viewdoc/ download?doi=10.1.1.550.6064\&rep=rep1\&type=pdf.

Hytrek, G. (1999), "Insurgent labour, economic change and social development: Costa Rica 1900-1948", Journal of Historical Sociology, vol. 12, No 1, Wiley.

Itzigsohn, J. (2000), Developing Poverty: The State, Labor Market Deregulation, and the Informal Economy in Costa Rica and the Dominican Republic, University Park, Pennsylvania, Pennsylvania State University Press.

Lehoucq, F. (2010), "Political competition, constitutional arrangements and the quality of public choices in Costa Rica", Latin American Politics and Society, vol. 52, № 4, Wiley.

(1998), Instituciones democráticas y conflictos políticos en Costa Rica, Heredia, Editorial Universidad Nacional Autónoma.

Lewis, C. y P. Lloyd-Sherlock (2009), "Social policy and economic development in South America: an historical approach to social insurance", Economy and Society, vol. 38, $N^{\circ} 1$, Taylor \& Francis.

Lizano, E. (1999), Ajuste y crecimiento en la economía de Costa Rica 1982-1994, San José, Academia de Centroamérica.

Martínez Franzoni, J. y D. Sánchez-Ancochea (2013a), Good Jobs and Social Services: How Costa Rica Achieved the Elusive Double Incorporation, Palgrave.

(2013b), "Can Latin American production regimes complement universalistic welfare regimes? Implications from the Costa Rican case", Latin American Research Review, vol. 48, N2 2, Asociación de Estudios Latinoamericanos (LASA).

(2012), "Inroads towards universalism: how Costa Rica informs theory", Documento de Trabajo, Notre Dame, Kellogg Institute for International Studies.

McGuire, J. (2010), Wealth, Health and Democracy in East Asia and Latin America, Nueva York, Cambridge University Press.

Mesa-Lago, C. (1978), Social Security in Latin America: Pressure Groups, Stratification and Inequality, Pittsburgh, University of Pittsburgh Press.

Mkandawire, T. (2006), "Targeting and universalism in poverty reduction", Social Policy and Development Programme Paper, Nㅜㄹ, Ginebra, Instituto de Investigaciones de las Naciones Unidas para el Desarrollo Social (UNRISD).

Monge González, R. y C. González-Vega (1995), Exportaciones tradicionales en Costa Rica, San José, Academia de Centroamérica.

Mortimore, M. y S. Vergara (2004), "Targeting winners: can foreign direct investment policy help developing countries industrialize?", European Journal of Development Research, vol. 16, Nㅜ 3, Taylor \& Francis.

Ocampo, J.A. y L. Taylor (1998), "Trade liberalisation in developing economies: modest benefits but problems with productivity growth, macro prices, and income distribution", Economic Journal, vol. 108, № 450, Wiley. 
OFIPLAN (Oficina de Planificación Nacional y Política Económica) (1982), Evolución socioeconómica de Costa Rica 1950-1980, San José, Editorial de la Universidad Estatal a Distancia (EUNED).

OIT (Organización Internacional del Trabajo) (2007), La igualdad en el trabajo: afrontar los retos que se plantean, Ginebra.

Pribble, J. (2011), "World apart: social policy regimes in Latin America", Studies in Comparative International Development, vol. 46, № 2, Springer.

Programa Estado de la Nación (2011), Estado de la nación en desarrollo humano sostenible. Decimoséptimo Informe, San José.

(2001), Estado de la nación en desarrollo humano sostenible. Séptimo informe, San José.

Reygadas, L. y F. Filgueira (2010), "Inequality and the incorporation crisis: the left's social policy toolkit", Latin America's Left Turns: Politics, Policies, and Trajectories of Change, C. Maxwell y E. Hershberg (eds.), Boulder, Lynne Rienner.

Rodríguez, C. (2007), "Estratificación y movilidad socio ocupacional en Costa Rica en la segunda mitad del siglo XX", Transformaciones en la estructura social en Costa Rica: estratos sociocupacionales, educación y trabajo, C. Castro y otros (eds.), San José, Instituto de Investigaciones Sociales.

(1997), "Los efectos del ajuste. Estratificación y movilidad ocupacional en Costa Rica en el período 19501995", tesis, Ciudad de México, El Colegio de México.

Rosemberg, M. (1983), Las luchas por el seguro social en Costa Rica, San José, Editorial Costa Rica.

Rovira, J. (2004), "El nuevo estilo de desarrollo nacional de Costa Rica 1984-2003 y el TLC", TLC con Estados Unidos: contribuciones para el debate. ¿Debe Costa Rica aprobarlo?, M.F. Flórez-Estrada y G. Hernández (eds.), San José, Universidad de Costa Rica.

(1990), "Costa Rica: elecciones, partidos políticos y régimen democrático", Polémica, № 11.

(1987), Costa Rica en los años 80, San José, Porvenir.

Rueschemeyer, D., E. Huber y J. Stephens (1992), Capitalist Development and Democracy, Chicago, University of Chicago Press.

Salazar, J.M. (1990), "El Estado y el ajuste en el sector industrial", Políticas económicas en Costa Rica, vol. 2, C. González Vega y E. Camacho Mejía (eds.), San José, Academia de Centroamérica.

Sánchez-Ancochea, D. (2009), "Are North-South trade agreements good for FDI-led development? The case of DR-CAFTA", Transnational Corporations and Development Policy: Critical Perspectives, E. Rugraff, D. Sánchez-Ancochea y A. Sumner (eds.), Londres, Palgrave Mcmillan.

(2003), "Globalization and inequality in the developing world: potential benefits with real costs", Nueva York, The New School.

Sandbrook, R. y otros (2007), Social Democracy in the Global Periphery: Origins, Challenges, Prospects, Cambridge, Cambridge University Press.

Schneider, A. (2012), State-building and Tax Regimes in Central America, Cambridge, Cambridge University Press.

Seekings, J. (2010), The ILO and Social Protection in the Global South, 1919-2005, Ginebra, Instituto Internacional de Estudios Laborales.

(2008), "Welfare regimes and redistribution in the South", Divide and Deal: The Politics of Distribution in Democracies, I. Shapiro, P. Swenson y D. Donno (ed.), Nueva York, New York University Press.

Segovia, A. (2005), Integración real y grupos de poder económico en América Central: Implicaciones para el desarrollo y la democracia de la región, San José, Fundación Friedrich Ebert.

Seligson, M. (1984), El campesino y el capitalismo agrario de Costa Rica, San José, Editorial de Costa Rica. (1980), Peasants of Costa Rica and the Development of Agrarian Capitalism, Wisconsin, University of Wisconsin Press.

Sojo, A. (1984), Estado empresario y lucha política en Costa Rica, San José, Editorial Universitaria Centroamericana.

Straface, F. (2008), "Gobernabilidad democrática en Costa Rica: ¿'hipergradualismo', cansancio reformista o desacuerdo sobre el modelo deseado?", Democracia estable ¿alcanza? Análisis de la gobernabilidad en Costa Rica, M. Gutiérrez Saxe y F. Straface (eds.), Washington, D.C., Banco Interamericano de Desarrollo (BID).

Taylor-Dormond, M. (1991), "El Estado y la pobreza en Costa Rica”, Revista de la CEPAL, № 43 (LC/G.1654-P), Santiago, Comisión Económica para América Latina y el Caribe (CEPAL).

Thorp, R. (1998), Progreso, pobreza y exclusión. Una historia económica de América Latina en el siglo XX, Washington, D.C., Banco Interamericano de Desarrollo (BID). 
Tokman, V. (2001), De la informalidad a la modernidad, Santiago, Organización Internacional del Trabajo (OIT). Torres-Rivas, E. (1975), Centroamérica hoy, Ciudad de México, Siglo XXI.

UNRISD (Instituto de Investigaciones de las Naciones Unidas para el Desarrollo Social) (2010), Combating Poverty and Inequality: Structural Change, Social Policy and Politics, Nueva York.

Vargas-Cullell, J., L. Rosero-Bixby y M. Seligson (2005), La cultura política de la democracia en Costa Rica, 2004, San José, Centro Centroamericano de Población (CCP) de la Universidad de Costa Rica.

Vega, M. (1982), El Estado costarricense de 1974 a 1978: CODESA y la fracción industrial, San José, Editorial Hoy.

(1980), "El Estado costarricense de 1974 a 1978. CODESA y la fracción industrial", tesis, San José, Universidad de Costa Rica.

Wilson, B. (1998), "When social democrats choose neoliberal economic policies: the case of Costa Rica", Comparative Politics, vol. 26, № 2.

Winson, A. (1989), Coffee and Democracy in Modern Costa Rica, Toronto, Between the Lines. 